\title{
Novel Transgenic Lines to Analyze Renal Glutathione Redox Potential In Vivo
}

\author{
Yuya Sugano, ${ }^{1,2}$ Hugo Siegfried, ${ }^{3}$ Erin Merkel, ${ }^{1,2}$ and lain A. Drummond ${ }^{1,2, *}$
}

\begin{abstract}
Reactive oxygen species (ROS) are important regulators of intracellular signaling pathways in health and disease. It is implicated that ROS may play critical roles in pathogenesis of a number of kidney diseases including diabetic nephropathy. However, due to the lack of tools for in vivo detection of redox status, our knowledge of redox dynamics is still fragmentary. In this study, we present novel zebrafish UAS transgenic lines expressing mitochondrial and cytoplasmic targeted redox fluorescent biosensors, Grx1roGFP2 and mitoGrx1-roGFP2. As the zebrafish is an ideal animal model for intravital imaging, these transgenic zebrafish provide useful tools to analyze renal redox dynamics in vivo.
\end{abstract}

Keywords: kidney, glutathione, ROS, roGFP2, mitochondria

$\mathbf{R}$ EDOX HOMEOSTASIS IS carefully controlled in biological systems. Dysregulation of redox balance results in accumulation of reactive oxygen species (ROS), causing deleterious cellular damage that potentially leads to disease and senescence. ${ }^{1}$ It has been increasingly recognized that ROS are central to pathogenesis of kidney disease. ${ }^{2}$ However, due to the lack of standardized tools to analyze the activity of this critical metabolic parameter in vivo, the role of ROS in kidney disease remains to be clearly defined. Glutathione is a major antioxidant that detoxifies ROS. Grx1-roGFP2, a ratiometric genetically encoded biosensor, reports the ratio of reduced glutathione and oxidized glutathione, thereby sensing the cellular redox state and oxidative stress levels. ${ }^{3}$

We generated transgenic zebrafish lines stably expressing Grx1-roGFP2 in the cytosol and mitoGrx1-roGFP2 in mitochondria under Gal4/UAS control [Tg(UAS:GrxlroGFP2) and $T g(U A S: m i t o G r x 1-r o G F P 2)]$ for flexible cell-type specific expression (Supplementary Data). Upon crossing with the Gal4 line $T g(c d h 17: G a l 4)$ driven by the pronephros-specific cadherin 17 ( $c d h 17)$ promoter,${ }^{4}$ the biosensors were specifically expressed in the pronephric tubules (Fig. 1A).

Grx1-roGFP2 biosensor dynamic range and responsiveness were tested by exposing transgenic embryos to a reducing agent reductant (dithiothreitol; DTT) or oxidant (tert butyl hydroperoxide; tBH) for 10 min (Fig. 1B, Supplementary Data). Ratiometric measurements of signals from 405 and $488 \mathrm{~nm}$ excitations demonstrated that the cytosol is in a reduced state, whereas mitochondria report a more oxidized state at rest (Fig. 1C). After 10 min exposure to an uncoupling agent, carbonilcyanide p-triflouromethoxyphenylhydrazone (FCCP), 405/488 nm excita-

\footnotetext{
${ }^{1}$ Nephrology Division, Department of Medicine, Massachusetts General Hospital, Boston, Massachusetts.

${ }^{2}$ Department of Genetics, Harvard Medical School, Boston, Massachusetts.

${ }^{3}$ Magistère Européen de Génétique, Université Paris Diderot, Paris, France.

*Current affiliation: Mount Desert Island Biological Laboratory, Bar Harbor, Maine.
} 
A
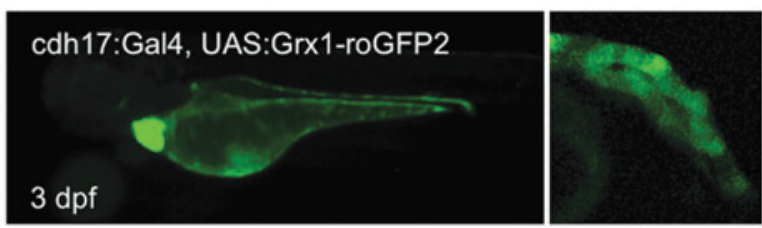

B

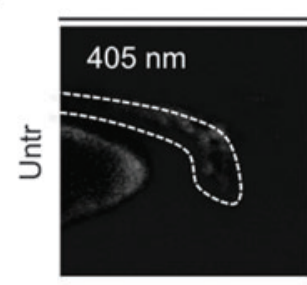

$\underset{\varpi}{\mathscr{\varpi}}$
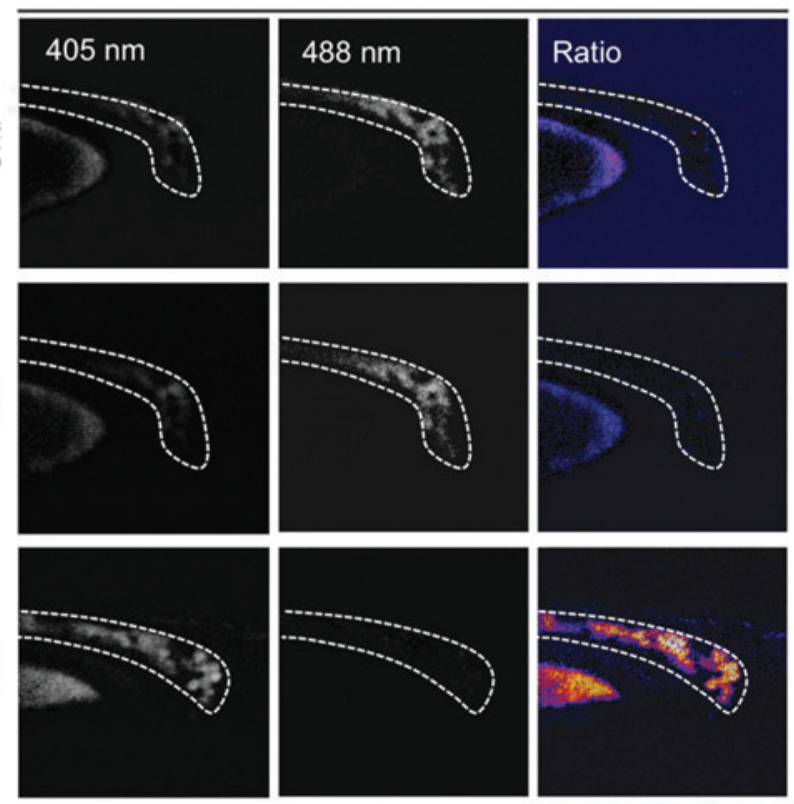

ช

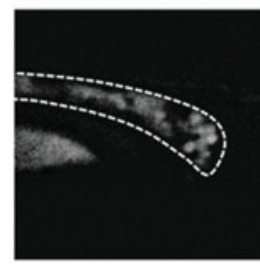

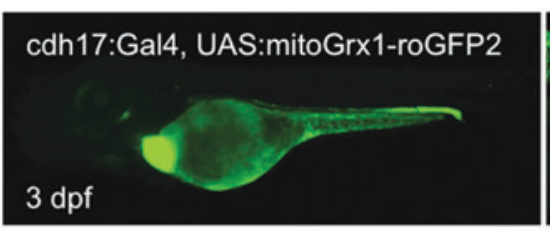

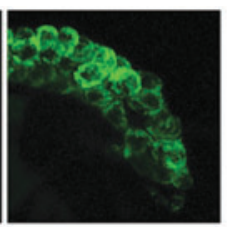

Mitochondrial
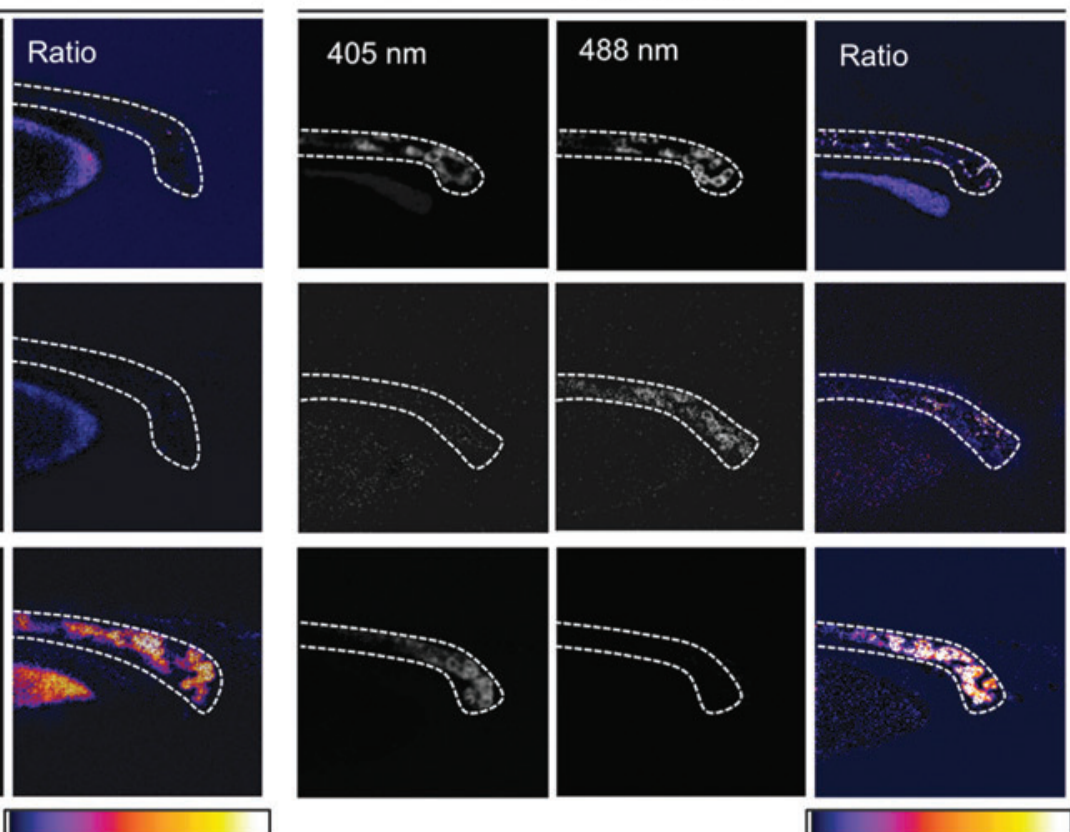

C
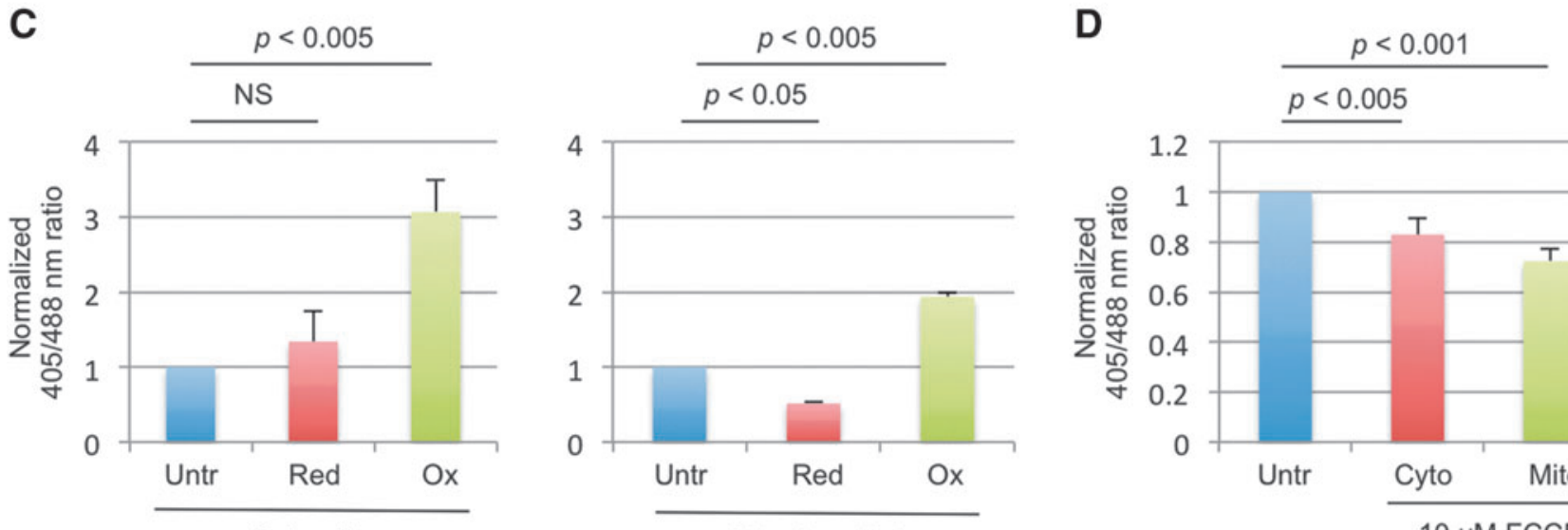

Cytosolic

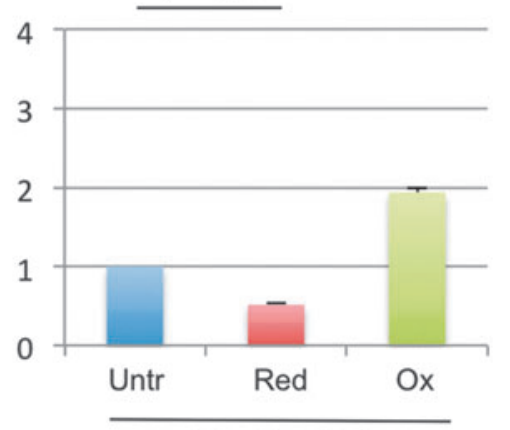

Mitochondrial

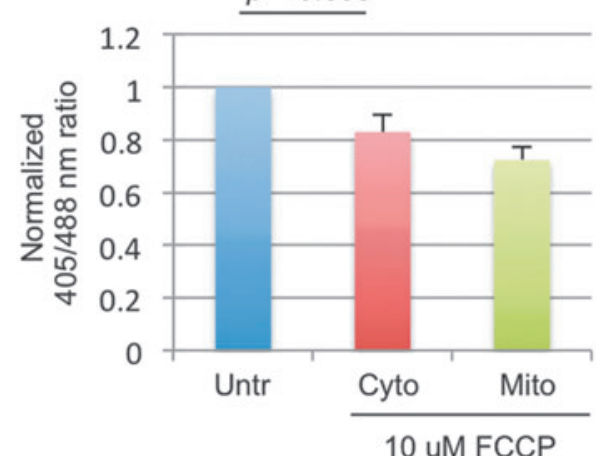

10 uM FCCP

FIG. 1. (A) Whole-mount fluorescent images of $\mathrm{Tg}(\mathrm{cdh} 17$ :Gal4, UAS:Grx1roGFP2) and $\operatorname{Tg}(\mathrm{cdh} 17: \mathrm{Gal} 4$, UAS:mitoGrx1-roGFP2) at 3 dpf. Higher magnification confocal images show the biosensor expression in the distal region of the pronephric tubules. (B) Both Grx1-roGFP2 and mitoGrx1-roGFP2 in tubular epithelia responded to reductant (DTT) and oxidant (tBH) as shown for the distal regions of the pronephros. (C) Normalized $405 / 488 \mathrm{~nm}$ ratios for fully reduced and fully oxidized states of Grx1-roGFP2 and mitoGrx1-roGFP2 are shown. The reduced and oxidized states $(t=10 \mathrm{~min})$ were each normalized to the untreated state of the same sample group ( $t=0 \mathrm{~min})$. Error bars represent the standard error. Cytosolic red: $n=8$, cytosolic ox: $n=10$, mitochondrial red: $n=8$, mitochondrial ox: $n=7$. (D) Ten minutes exposure of the transgenic embryos to $10 \mu \mathrm{M}$ FCCP led to decreased glutathione redox potential both in the cytosol and in mitochondria. $n=10$ both for cytosolic and mitochondrial. $p$-values were obtained from Student's $t$-test. dpf, days postfertilization; DTT, dithiothreitol; FCCP, carbonilcyanide p-triflouromethoxyphenylhydrazone; tBH, tert butyl hydroperoxide. 
tion ratios of the biosensors decreased both in the cytosol and mitochondria, indicating that uncoupling of oxidative phosphorylation from adenosine triphosphate (ATP) synthesis reduces cellular oxidative stress levels (Fig. 1D). Uncoupling enhances mitochondrial oxygen consumption, presumably leading to decrease in the formation of oxygen radicals. ${ }^{5}$ As our approach is applicable to other cell types and tissues by using different Gal4 driver lines, the transgenic zebrafish in this study represent powerful and versatile tools to investigate redox dynamics in living animals.

\section{Disclosure Statement}

Y.S., H.S., E.M., and I.A.D. have no commercial associations to report and no conflicts of interest in connection with this manuscript.

\section{Funding Information}

Y.S. was funded by Swiss National Science Foundation Early/Advanced Postdoc Mobility Fellowship. The project was supported by NIH grants DK053093 to I.A.D. and the NIDDK Diabetic Complications Consortium (RRID: SCR_001415, www .diacomp.org), grants DK076169 and DK115255.

\section{Supplementary Material}

Supplementary Data

\section{References}

1. Sivandzade F, Prasad S, Bhalerao A, Cucullo L. NRF2 and NF-B interplay in cerebrovascular and neurodegenerative disorders: molecular mechanisms and possible therapeutic approaches. Redox Biol 2019;21:101059.

2. Forbes JM, Thorburn DR. Mitochondrial dysfunction in diabetic kidney disease. Nat Rev Nephrol 2018;14:291-312.

3. Gutscher M, Pauleau AL, Marty L, Brach T, Wabnitz GH, Samstag Y, et al. Real-time imaging of the intracellular glutathione redox potential. Nat Methods 2008;5:553-559.

4. Zhou W, Boucher RC, Bollig F, Englert C, Hildebrandt F. Characterization of mesonephric development and regeneration using transgenic zebrafish. Am J Physiol Renal Physiol 2010;299:F1040-F1047.

5. Skulachev VP. Uncoupling: new approaches to an old problem of bioenergetics. Biochim Biophys Acta 1998;1363:100-124.

Address correspondence to: Iain A. Drummond, PhD Mount Desert Island Biological Laboratory Bar Harbor, ME 04609

E-mail: idrummond@mdibl.org 